\title{
Consolidation of Human Motor Cortical Neuroplasticity by D-Cycloserine
}

\author{
Michael A Nitsche*,', Wiebke Jaussi', David Liebetanz', Nicolas Lang', Frithjof Tergau' and Walter Paulus' \\ 'Department Clinical Neurophysiology, Georg-August-University, Goettingen, Germany
}

\begin{abstract}
D-Cycloserine (CYC), a partial N-methyl-D-aspartate (NMDA) agonist, has been shown to improve cognitive functions in humans. However, the neurophysiological basis of this effect is unclear so far. We studied the impact of this drug on long-lasting after-effects of transcranial direct current (tDCS)-generated motor cortical excitability shifts, as revealed by transcranial magnetic stimulation-elicited motor-evoked potentials. While anodal tDCS enhances motor cortical excitability, cathodal tDCS diminishes it. Both effects seem to be NMDA receptor dependent. D-CYC selectively potentiated the duration of motor cortical excitability enhancements induced by anodal tDCS. D-CYC alone did not modulate excitability. The potency of this drug to consolidate neuronal excitability enhancements, most probably by stabilizing the strengthening of NMDA receptors, which is a probable neurophysiological derivate of learning processes, makes it an interesting substance to improve cognitive functions.

Neuropsychopharmacology (2004) 29, 1573-1578, advance online publication, 16 June 2004; doi: 10.1038/sj.npp. 1300517
\end{abstract}

Keywords: neuroplasticity; transcranial magnetic stimulation; transcranial direct current stimulation; human; brain; D-cycloserine; motor cortex; cognition

\section{INTRODUCTION}

D-Cycloserine (CYC) was initially introduced as a tuberculostatic agent (Walker and Murdoch, 1957). A number of side effects of this drug results from its penetration into the central nervous system. Here, interestingly, it functions as a selective partial $N$-methyl-D-aspartate (NMDA) agonist. CYC acts at the glycine-binding site of the NMDA receptor, thus facilitating the opening of the NMDA channel (Thomas et al, 1988). In slice preparations, it has been shown that the activation of this subunit is of importance for inducing long-term potentiation (LTP) effects, and that CYC can enhance LTP (Watanabe et al, 1992). The effect of CYC is thereby dosage dependent. It acts as a positive modulator at the NMDA receptor at low dose, but as a negative modulator at high dose (Anthony and Nevins, 1993). LTP is an important neuronal substrate of learning, at least in animals (Rioult-Pedotti et al, 2000). Therefore, studies were recently conducted to explore if the administration of CYC could improve cognitive functions in humans. Indeed, in some studies, it has been shown to improve cognitive functions in Alzheimer and schizophrenic patients, if

\footnotetext{
*Correspondence: Dr MA Nitsche, Department Clinical Neurophysiology, Georg-August-University, Robert-Koch-Str. 40, Goettingen 37075, Germany, Tel: + 49 55I 39 12631, Fax: + 49 55I 39 8126, E-mail: mnitsch I@gwdg.de

Received 27 December 2003; revised 30 March 2004; accepted 20 May 2004

Online publication: 2I May 2004 at http://www.acnp.org/citations/ Npp05210403578/default.pdf
}

administered in a proper dosage (Goff et al, 1995, 1999; Tsai et al, 1998, 1999), although the results achieved so far are mixed (Laake and Oeksengaard, 2002). Whether the improvement of cognitive functions is caused by an enhancement of NMDA receptor efficacy has so far not been tested in humans.

Interestingly, in Alzheimer's disease the NMDA receptor antagonist memantine was also shown to improve cognitive functions. The beneficial effect of both drugs is probably due to different mechanisms of action with regard to NMDA receptor activity. While CYC seems to enhance primarily phasic receptor activation and its consequences, that is, LTP, memantine is thought to act on tonic receptor activity. It is speculated that it could thereby enhance cognitive functions - on the one hand, by increasing the synaptic signal-to-noise ratio in advance for phasic, learning-related, synaptic activity, and on the other, reduce excitotoxic effects triggered by glutamatergic activity (Danysz and Parsons, 2003; Rogawski and Wenk, 2003). Moreover, memantine is thought to protect neurons against the damaging effects of $\beta$-amyloid (Miguel-Hidalgo et al, 2002).

The transcranial administration of weak direct currents (tDCS) results in excitability changes of motor and visual cortices in the human, which evolve during tDCS but outlast the stimulation for up to an hour, given a sufficiently long duration of stimulation. Anodal tDCS increases, while cathodal tDCS decreases excitability (Antal et al, 2003; Nitsche and Paulus, 2000, 2001; Nitsche et al, 2003a). Moreover, the technique is functionally relevant: for the motor cortex, it has been shown to modulate use-dependent 
neuroplasticity (Rosenkranz et al, 2000) and to improve implicit motor learning (Nitsche et al, 2003b). The aftereffects are of intracortical origin. Whereas the initial effect of DC stimulation is accomplished by a hyper- or depolarization of neuronal membranes (Nitsche et al, 2003c; Purpura and McMurtry, 1965), the after-effects seem to be NMDA receptor dependent (Liebetanz et al, 2002; Nitsche et al, 2003c). However, since the involvement of NMDA receptors in the tDCS-elicited after-effects was so far tested solely by the administration of dextrometorphan, an NMDA receptor antagonist that also has ion channel blocking capabilities (Netzer et al, 1993), no definite conclusions about the involvement of these receptors in the after-effects are currently possible.

The aim of this study was to test directly if CYC influences tDCS-induced cortical neuroplasticity. Therefore, we administered tDCS protocols known to induce prolonged cortical excitability shifts to the CYC-conditioned human brain. In addition, the effect of CYC alone on corticospinal and intracortical excitability was tested to prove the selectivity of its effects on ongoing neuroplastic processes.

We chose the motor cortex as a model for NMDA-related cortical plasticity because LTP and depression, as well as use-dependent plasticity in this cortex, are NMDA receptor dependent (Castro-Alamancos et al, 1995; Hess et al, 1996; Bütefisch et al, 2000) and the involvement of LTP in learning processes has been demonstrated for it (RioultPedotti et al, 2000). Moreover, in contrast to the hippocampus, which is more frequently used in slice preparations to study neuroplasticity, external stimulation techniques are more easily applicable to this area due to its topological position.

We administered a relatively low dosage of $100 \mathrm{mg}$ CYC. According to animal experiments (Anthony and Nevins, 1993), CYC at this dosage should modulate the NMDA receptor positively. Moreover, CYC at this dosage has been shown to be beneficial in Alzheimer's disease (Tsai et al, 1999). We hypothesized that CYC would augment the anodal tDCS-elicited cortical excitability enhancement because of the proposed synergistic action of tDCS and CYC on NMDA receptors. Conversely, we did not expect an effect of CYC on the cathodal tDCS-driven excitability diminution. We think that these are produced by a presynaptic reduction of glutamate release due to reduced neuronal activity, as shown directly in animals (Bindman et al, 1964), and a postsynaptic membrane hyperpolarization. These factors act in combination to induce long-term excitability diminutions in animals (Frégnac et al, 1990). In this case, a moderate dosage of CYC should not suffice to counteract the tDCS-induced membrane effects on NMDA receptor activity. Moreover, since CYC primarily does facilitate, but not induce NMDA channel opening, it was thought not to modify the effects of low-frequency synaptic input on NMDA receptor strength relevantly.

\section{METHODS}

\section{Subjects}

In all, 12 healthy subjects participated in the experiments (mean age 26.33 years (3.58 SD (standard deviation), five male). All gave written informed consent. The investigation was approved by the ethics committee of the University of Goettingen, and we confirm to the Declaration of Helsinki.

\section{Current Stimulation of the Motor Cortex}

Direct currents were transferred by a saline-soaked pair of surface sponge electrodes $\left(35 \mathrm{~cm}^{2}\right)$ and delivered by a specially developed battery-driven constant current stimulator (Schneider Electronic, Gleichen, Germany) with a maximum output of $2 \mathrm{~mA}$. The motor cortical electrode was fixed over the representational field of the right abductor digiti minimi (ADM) as identified by transcranial magnetic stimulation (TMS) and the other electrode contralaterally above the right orbit. tDCS was applied for $9 \mathrm{~min}$ (cathodal tDCS, polarity refers to the motor cortical electrode) and 13 min (anodal tDCS) with an intensity of $1.0 \mathrm{~mA}$. These stimulation protocols were chosen to induce after-effects lasting about $1 \mathrm{~h}$, as have been achieved by these stimulation durations in previous experiments (Nitsche and Paulus, 2001; Nitsche et al, 2003a). Nearly all subjects were able to feel the current flow as a slight itching sensation at both the anodal and cathodal electrodes.

\section{Pharmacological Interventions}

With regard to Experiment 1, $100 \mathrm{mg}$ CYC or equivalent placebo (PLC) drugs were administered to the subjects orally $2 \mathrm{~h}$ before the start of each experimental session. At $2 \mathrm{~h}$ after oral intake, CYC induces a stable plasma level (van Berckel et al, 1997). For Experiment 2, CYC was administered immediately after a baseline measurement of intracortical and corticospinal excitability, the second measurement was conducted $2 \mathrm{~h}$ after intake. To avoid cumulative drug effects, each experimental session was separated by at least 1 week. Subjects were blinded to the respective pharmacological condition.

\section{Measurement of Motor Cortical Excitability}

Experiment 1. To detect current-driven changes of excitability, motor-evoked potentials (MEPs) of the right ADM were recorded following stimulation of its motor cortical representational field by single pulse TMS. These were elicited by single-pulse TMS using a Magstim 200 magnetic stimulator (Magstim Company, Whiteland, Dyfed, UK) and a figure-ofeight magnetic coil (diameter of one winding $=70 \mathrm{~mm}$, peak magnetic field $=2.2 \mathrm{~T}$ ). The coil was held tangentially to the skull, with the handle pointing backwards and laterally at $45^{\circ}$ from midline. The optimal position was defined as the site where stimulation resulted consistently in the largest motorevoked potential. Surface EMG was recorded from the right ADM. The signals were amplified and filtered with a time constant of $10 \mathrm{~ms}$ and a low-pass filter of $2.5 \mathrm{kHz}$. Signals were then digitalized at an analogue-to-digital rate of $5 \mathrm{kHz}$, and further relayed into a laboratory computer using the Neuroscan software collection (Neuroscan Inc., Herndon, VA, USA) and conventional averaging software.

Experiment 2. The equipment used for this experiment was identical to Experiment 1. Intracortical inhibition and facilitation was measured by the Kujirai TMS double stimulation protocol including 2, 3, 5, 10, and $15 \mathrm{~ms}$ 
interstimulus intervals (ISI), the first two ISIs representing inhibitory and the last two ISIs facilitatory intervals (Kujirai et al, 1993). In this protocol, a subthreshold conditioning stimulus precedes a suprathreshold test stimulus. The stimuli were organized in blocks: in each block, each double pulse was included one time, added by a single test pulse. The blocks were repeated 15 times, the order of the different pulses was randomized between blocks. The intensity of the conditioning pulse was $90 \%$ of active motor threshold. Active motor threshold was defined as the lowest TMS intensity resulting in an MEP higher in amplitude than moderate spontaneous muscular activity in at least three out of six trials. Single test pulse stimulation intensity was adjusted to achieve a baseline MEP of about $1 \mathrm{mV}$.

\section{Experimental Procedures}

Experiment 1. Each experiment was conducted in a repeated measurement design. The subjects were seated in a reclining chair. First, the left motor cortical representational field of the right ADM was identified by use of TMS (coil position that leads to the largest MEPs of ADM). Then, one DC stimulation electrode was fixed at this position and the other one at the contralateral forehead above the orbit.

First, a baseline of TMS-evoked MEPs (20 stimuli) was recorded at $0.25 \mathrm{~Hz}$. Afterwards anodal $(13 \mathrm{~min}$ ) or cathodal (9 min) tDCS was administered to produce long-lasting after-effects. After termination of tDCS, 15 MEPs were recorded at $0.25 \mathrm{~Hz}$ every fifth minute up to $30 \mathrm{~min}$, then every half hour up to 90 min (cathodal tDCS/CYC and PLC conditions) or $120 \mathrm{~min}$ (anodal tDCS/CYC). For the anodal condition, these measurements were repeated at $240 \mathrm{~min}$ after stimulation as well as in the morning and the afternoon of the poststimulation day. The electrode and coil positions were marked with a water-proof pen to guarantee identical positions during the whole course of the experiment. The respective tDCS/drug condition-dependent protocols differ with respect to the duration of the aftereffect recording in accordance with former experiments and the hypotheses about the proposed tDCS/CYC interactions outlined in the introduction. Under PLC conditions, the after-effects of tDCS should have vanished $90 \mathrm{~min}$ after tDCS (Nitsche and Paulus, 2001; Nitsche et al, 2003a). As we did not expect a prolongation of the cathodal tDCS-induced after-effects through administration of CYC, measurement was also terminated here $90 \mathrm{~min}$ after tDCS. For the anodal tDCS/CYC condition, we expected a relevant prolongation of the after-effects, so those from this condition were recorded until the afternoon of the poststimulation day.

Experiment 2. Here, after determining active motor threshold of the ADM, first a baseline measure of intracortical excitability was performed, consisting of 15 blocks of the above-mentioned double stimuli with a frequency of $0.25 \mathrm{~Hz}$, and then CYC was administered. After $2 \mathrm{~h}$, active motor threshold was determined again, and then the identical double stimulation protocol was repeated.

\section{Calculations and Statistics}

Experiment 1. MEP amplitude means were calculated for each time bin covering baseline (20 stimuli) and post- stimulation time points (15 stimuli). These were normalized to precurrent baselines. Repeated measures ANOVAs (independent variables time course, current stimulation, drug condition, dependent variable MEP amplitude) were calculated for the first 90 min after tDCS (which were covered by the anodal and cathodal measures), then Student's $t$-tests (paired samples, two-tailed, level of significance $<0.05$ ) were performed to test whether the MEP amplitudes before and after tDCS differed in each condition, and if those differences were dependent on the drug conditions. Post hoc $t$-tests were not corrected for multiple comparisons according to Perneger (1998).

Experiment 2. Student's $t$-tests (two-tailed, paired samples) were calculated for baseline and post-CYC active thresholds and single-pulse MEP amplitudes to look for changes caused by CYC intake.

Intraindividual MEP amplitude means were calculated for each ISI before and after CYC intake. The resulting means were standardized to the single-pulse condition. A repeated measures ANOVA (independent variables: ISI and time point relative to CYC intake; dependent variable: MEP amplitude) was calculated.

\section{RESULTS}

\section{Experiment 1}

The results of the ANOVA (Table 1) show significant main effects of tDCS and time course, the main effect of drug was not significant. Furthermore, the interactions between tDCS $\times$ time course, drug $\times$ time course, as well as tDCS $\times$ drug $\times$ time course were significant. As demonstrated by the post hoc $t$-tests, this pattern of results is due to a significant MEP amplitude diminution lasting for an hour after cathodal tDCS, irrespective of drug condition

Table I ANOVAs Covering the Effects of CYC on tDCSGenerated Excitability Changes and the Effects of CYC Alone on Cortical Excitability

\begin{tabular}{|c|c|c|c|}
\hline & df & $\mathbf{F}$ & $p$-value \\
\hline \multicolumn{4}{|l|}{ Experiment I } \\
\hline tDCS & । & 108.174 & $<0.00$ I* \\
\hline Drug & 1 & 0.248 & 0.628 \\
\hline Time course & 9 & 2.287 & $0.022 *$ \\
\hline tDCS $\times$ drug & 1 & 0.741 & 0.408 \\
\hline tDCS $\times$ time course & 9 & 25.971 & $<0.00$ I* \\
\hline Drug $\times$ time course & 9 & 5.836 & $<0.00$ I* \\
\hline tDCS $\times$ drug $\times$ time course & 9 & 3.695 & $0.001 *$ \\
\hline \multicolumn{4}{|l|}{ Experiment 2} \\
\hline$|S|$ & 4 & 278.978 & $<0.00$ I* \\
\hline Time point & 1 & 0.324 & 0.583 \\
\hline$|S| \times$ Time point & 4 & 0.422 & 0.532 \\
\hline
\end{tabular}

Results of the ANOVAs with regard to the long-term effects of tDCS under D-CYC and placebo medications (Experiment I) and the effect of D-CYC on intracortical excitability (Experiment 2) are shown. Note that CYC interacts selectively with the time course and tDCS condition, but does not modulate intracortical excitability alone. Asterisks indicate significant results. 
(PLC, $p$ between 0.001 and $<0.001$; CYC, $p<0.001$ in all conditions), and by a significant excitability enhancement caused by anodal tDCS lasting for an hour after stimulation with regard to the PLC condition ( $p$ between 0.001 and 0.009 ), while remaining stable until the next morning after CYC administration ( $p$ between 0.018 and $<0.001$ in all conditions; Figure 1). With regard to anodal tDCS, MEP amplitudes did differ between the PLC and CYC condition in the measurement conducted $90 \mathrm{~min}$ after tDCS $(p<0.001)$. Under CYC, MEP amplitudes were still enhanced, while they were at baseline level in the PLC condition. For the cathodal stimulation condition, CYC caused a significantly increased excitability diminution compared to PLC only $10 \mathrm{~min}$ after tDCS $(p=0.001)$. The first measure at the day after CYC intake was performed between 17 and $23 \mathrm{~h}$, the second one between 22 and $29 \mathrm{~h}$ after drug intake.

Baseline mean MEP values in each drug/tDCS condition were between 971.5 and $1008.2 \mu \mathrm{V}$ in each condition and did not differ significantly from each other (two-tailed paired $t$ tests, $p>0.05)$. Apart from a slight itching under the tDCS electrodes, no subject reported any side effect of the stimulation or drug intake.

\section{Experiment 2}

MEP amplitudes elicited by a single TMS pulse did not differ before and $2 \mathrm{~h}$ after CYC intake (Figure 2). Also active motor thresholds were identical in the two conditions (twotailed paired i-tests, $p>0.05$ ).

With regard to the double stimulation paradigm, the ANOVA revealed a significant main effect of ISI, but time point as well as the interaction between time point and ISI were not significant (Figure 2).

\section{DISCUSSION}

In this study, we tested the impact of the partial NMDA receptor agonist CYC on externally induced motor cortical

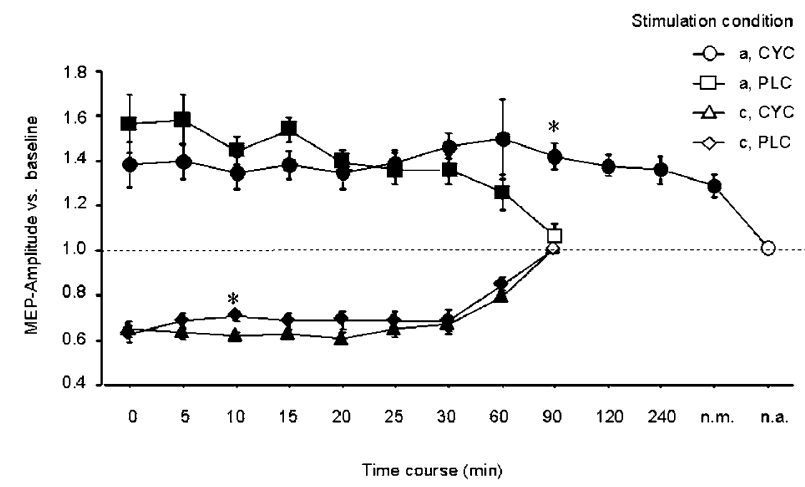

Figure I D-CYC selectively enhances the duration of the anodal tDCSinduced excitability enhancement, whereas the duration of the cathodal tDCS-induced excitability diminution is left unchanged. Filled symbols indicate significant alterations of the post-tDCS MEP amplitudes from the baseline values, asterisks indicate significant differences between PLC and CYC conditions at one time point with regard to anodal or cathodal tDCS. Error bars show standard error of means (SEM). n.m. = next morning; n.a. $=$ next afternoon.

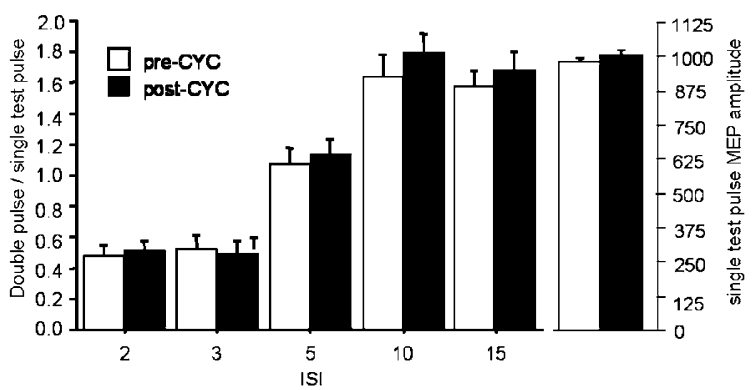

Figure 2 CYC does not change intracortical excitability significantly, as shown by identical MEP amplitudes elicited by TMS double stimulation for inhibiting and facilitating ISIs with and without CYC. Also corticospinal excitability is not influenced by CYC alone, as shown by identical single pulse TMS-elicited MEP amplitudes with and without CYC (right part of the figure). Error bars show SEM.

excitability shifts generated by tDCS. CYC selectively prolonged-but did not increase-the anodal tDCS-induced excitability enhancement. As compared to the PLC condition, it lasted relevantly longer under CYC. The fact that MEP amplitudes under CYC were still enhanced the morning after stimulation, as compared to baseline values, suggests a prolongation of the excitability enhancement for several hours as compared to the PLC condition, especially since MEPs have been shown to be relatively stable over time (Ziemann et al, 1996; Strutton et al, 2003). However, since we did not measure MEPs under PLC medication for the same duration, definite conclusions about the exact duration of the prolongation of the after-effects by CYC cannot be derived from the results of this study. Conversely, the excitability diminution elicited by cathodal tDCS was not relevantly influenced by prior CYC administration.

Since the after-effects of tDCS are suggested to be NMDA receptor dependent (Liebetanz et al, 2002), the prolongation of the excitability enhancement is most probably due to a modulating effect of the partial NMDA receptor agonist CYC. By binding at the glycine receptor site, CYC could enhance the efficacy of anodal tDCS-induced NMDA receptor activation. In animals, it has been shown directly that CYC is able to facilitate NMDA receptor activity (Thomas et al, 1988). We propose that the positive effect of CYC on NMDA receptors increases the level of tDCSinduced long-term excitability elevations and, thus, may help to 'memorize' and extend the anodal tDCS-induced after-effects.

Conversely, the lack of an effect in case of the cathodal tDCS-induced excitability diminution can be explained by a reduction of NMDA receptor activity caused by tDCS, which would not be antagonized by a partial activation of its glycine-binding site alone. This would also explain the - at first sight surprising-difference between the results obtained in this study and those reported after the administration of dextrometorphan, an NMDA receptor antagonist. This drug eliminated the after-effects of anodal and of cathodal tDCS (Liebetanz et al, 2002; Nitsche et al, 2003c). While an increase of after-effects under CYC and a decrease under dextromethorphan, which was observed in the anodal tDCS condition, seems plausible, if the tDCSdependent excitability changes are NMDA receptor dependent, the absent effect of CYC in the cathodal tDCS 
condition requires some further explanation. We suggest that cathodal tDCS weakens NMDA receptors by reducing their activity via decreasing, but not abolishing, the afferent input and hyperpolarizing the postsynaptic membrane. Reduced cortical activity and membrane hyperpolarization have been shown to be induced by cathodal DC stimulation directly in animals (Bindman et al, 1964, Purpura and McMurtry, 1965). Moreover, a combination of both mechanisms has been demonstrated to induce long-lasting excitability diminutions in animals (Frégnac et al, 1990). An NMDA receptor block, which is produced by dextrometorphan, would abolish the effects of the low-frequency input on the activity of the NMDA receptor and, thus, eliminate the after-effects induced by cathodal tDCS. Conversely, a partial NMDA receptor agonist, applied at a dosage, which modulates the receptor positively, but only facilitates channel opening, would leave both mechanisms intact and, thus, not modify the after-effects. This is exactly the result observed in this study. However, further studies are required to clarify the action of tDCS on NMDA receptors.

Interestingly, CYC alone had no significant effect on corticospinal or on intracortical excitability, as shown by identical MEP amplitudes of single and double TMS pulses with and without CYC.

The results of this study may explain why CYC has a certain potential to improve learning processes in animals (Baxter et al, 1994; Pitkanen et al, 1995) as well as in humans (Tsai et al, 1999). NMDA receptors have been shown to be involved in learning processes (Hauben et al, 1999; Newcomer and Krystal, 2001; Donchin et al, 2002). Moreover, it is proposed that NMDA receptor-dependent LTP serves as one neurophysiological correlate of learning and memory formation (Rioult-Pedotti et al, 1998). Thus, it may be speculated that CYC improves cognitive functions by enhancing the efficacy of glutamatergic transmission via stabilization of the strengthening of NMDA receptors during learning processes.

Taken together, by its ability to consolidate NMDA receptor efficacy enhancements, CYC is a potentially interesting substance to enhance cognitive functions. More studies to test its efficacy in cognitive tasks as well as systematic dose-finding studies are needed to test its relevance for improving learning processes in healthy subjects as well as in patients with disturbed cognitive functions.

\section{REFERENCES}

Antal A, Kincses TZ, Nitsche MA, Paulus W (2003). Manipulation of phosphene thresholds by transcranial direct current stimulation in man. Exp Brain Res 150: 375-378.

Anthony EW, Nevins ME (1993). Anxiolytic-like effects of $\mathrm{N}$ methyl-D-aspartate-associated glycine repeptor ligands in the rat potentiated startle test. Eur J Pharmacol 250: 317-324.

Baxter MG, Lanthorn TH, Frick KM, Golski S, Wan RQ, Olton DS (1994). D-Cycloserine, a novel cognitive enhancer, improves spatial memory in aged rats. Neurobiol Aging 15: 207-213.

Bindman LJ, Lippold OC, Redfearn JW (1964). The action of brief polarizing currents on the cerebral cortex of the rat (1) during current flow and (2) in the production of long-lasting aftereffects. J Physiol 172: 369-382.

Bütefisch CM, Gambetti P, Davis BC, Wise SP, Sawaki L, Kopylev L et al (2000). Mechanisms of use-dependent plasticity in the human motor cortex. Proc Natl Acad Sci USA 97: 33661-33665.

Castro-Alamancos MA, Donoghue JP, Connors BW (1995). Different forms of synaptic plasticity in somatosensory and motor areas of the neocortex. J Neurosci 15: 5324-5333.

Danysz W, Parsons CG (2003). The NMDA receptor antagonist memantine as a symptomatological and neuroprotective treatment for disease: preclinical evidence. Int J Geriatr Psychiatry 18: S23-S32.

Donchin O, Sawaki L, Madupu G, Cohen LG, Shadmehr L (2002). Mechanisms influencing acquisition and recall of motor memories. J Neurophysiol 88: 2114-21123.

Frégnac Y, Smith D, Friedlander MJ (1990). Postsynaptic membrane potential regulates synaptic potentiation and depression in visual cortical neurons. Soc Neurosci Abstr 16: 798.

Goff DC, Henderson DC, Evins AE, Amico E (1999). A placebocontrolled crossover trial of D-cycloserine added to clozapine in patients with schizophrenia. Biol Psychiatry 45: 512-514.

Goff DC, Tsai G, Manoach DS, Coyle JT (1995). Dose-finding trial of D-cycloserine added to neuroleptics for negative symptoms in schizophrenia. Am J Psychiatry 152: 1213-1215.

Hauben U, D’Hooge R, Soetens E, De Deyn PP (1999). Effects of oral administration of the competitive $N$-methyl-D-asparate antagonist, CGP 40116, on passive avoidance, spatial learning, and neuromotor abilities in mice. Brain Res Bull 48: 333-341.

Hess G, Aizenman CD, Donoghue JP (1996). Conditions for the induction of long-term potentiation in layer II/III horizontal connections of the rat motor cortex. J Neurophysiol 75: $1765-1778$.

Kujirai T, Caramia MD, Rothwell JC, Day BL, Thompson PD, Ferbert A et al (1993). Corticocortical inhibition in human motor cortex. J Physiol 471: 501-519.

Laake K, Oeksengaard AR (2002). D-Cycloserine for Alzheimer's disease. Cochrane Database Syst Rev 2: CD003153.

Liebetanz D, Nitsche MA, Tergau F, Paulus W (2002). Pharmacological approach to synaptic and membrane mechanisms of DCinduced neuroplasticity in man. Brain 125: 2238-2247.

Miguel-Hidalgo JJ, Alvarez XA, Cacabelos R, Quack G (2002). Neuroprotection by memantine against neurodegeneration induced by beta-amyloid (1-40). Brain Res 958: 210-221.

Netzer R, Pflimlin P, Trube G (1993). Dextromethorphan blocks $N$ methyl-D-aspartate-induced currents and voltage-operated inward currents in cultured cortical neurons. Eur J Pharmacol 238: 209-216.

Newcomer JW, Krystal JH (2001). NMDA receptor regulation of memory and behavior in humans. Hippocampus 11: 529-542.

Nitsche MA, Fricke K, Henschke U, Schlitterlau A, Liebetanz D, Lang $\mathrm{N}$ et al (2003c). Pharmacological modulation of membrane potential and NMDA receptor efficacy shifts during and after transcranial weak direct current stimulation of the human motor cortex. J Physiol 553: 293-301.

Nitsche MA, Nitsche MS, Klein CC, Tergau F, Rothwell JC, Paulus W (2003a). Level of action of cathodal DC polarisation induced inhibition of the human motor cortex. Clin Neurophysiol 114: 600-604.

Nitsche MA, Paulus W (2000). Excitability changes induced in the human motor cortex by weak transcranial direct current stimulation. J Physiol 527: 633-639.

Nitsche MA, Paulus W (2001). Sustained excitability elevations induced by transcranial DC motor cortex stimulation in humans. Neurology 57: 1899-1901.

Nitsche MA, Schauenburg A, Lang N, Liebetanz D, Exner C, Paulus $\mathrm{W}$ et al (2003b). Facilitation of implicit motor learning by weak transcranial direct current stimulation of the primary motor cortex in the human. J Cogn Neurosci 15: 619-626.

Perneger TV (1998). What's wrong with Bonferroni adjustments. BMJ 316: 1235-1238. 
Pitkanen M, Sirvio J, MacDonald E, Niemi S, Ekonsalo T, Riekkinen Sr P (1995). The effects of D-cycloserine and MK801 on the performance of rats in two spatial learning and memory tasks. Eur Neuropsychopharmacol 5: 457-463.

Purpura DP, McMurtry JG (1965). Intracellular activities and evoked potential changes during polarization of motor cortex. J Neurophysiol 28: 166-185.

Rioult-Pedotti MS, Friedman D, Donoghue JP (2000). Learninginduced LTP in neocortex. Science 290: 533-536.

Rioult-Pedotti MS, Friedman D, Hess G, Donoghue JP (1998). Strengthening of horizontal cortical connections following skill learning. Nat Neurosci 1: 230-234.

Rogawski MA, Wenk GL (2003). The neuropharmacological basis for the use of memantine in the treatment of Alzheimer's disease. CNS Drug Rev 9: 275-308.

Rosenkranz K, Nitsche MA, Tergau F, Paulus W (2000). Diminution of transient motor cortex plasticity by weak transcranial direct current stimulation in the human. Neurosci Lett 296: 61-63.

Strutton PH, Catley M, Davey NJ (2003). Stability of corticospinal excitability and grip force in intrinsic hand muscles in man over a 24-h period. Physiol Behav 79: 679-682.
Thomas JW, Hood WF, Monahan JB, Contreras PC, O'Donohue TL (1988). Glycine modulation of the phencyclidine binding site in mammalian brain. Brain Res 442: 396-398.

Tsai GE, Falk WE, Gunther J (1998). A preliminary study of Dcycloserine treatment in Alzheimer's disease. J Neuropsychiatry Clin Neurosci 10: 224-226.

Tsai GE, Falk WE, Gunther J, Coyle JT (1999). Improved cognition in Alzheimer's disease with short-term D-cycloserine treatment. Am J Psychiatry 156: 467-469.

van Berckel BN, Lipsch C, Timp S, Gispen-de Wied C, Wynne H, van Ree JM, Kahn RS (1997). Behavioral and neuroendocrine effects of the partial NMDA agonist D-cycloserine in healthy subjects. Neuropsychopharmacology 16: 317-324.

Walker JC, Murdoch JM (1957). Cycloserine in the treatment of pulmonary tuberculosis. A report on toxicity. Tubercle 38: 297.

Watanabe Y, Saito H, Abe K (1992). Effects of glycine and structurally related amino acids on generation of long-term potentiation in rat hippocampal slices. Eur J Pharmacol 223: 179-184.

Ziemann U, Lonnecker S, Steinhoff BJ, Paulus W (1996). Effects of antiepileptic drugs on motor cortex excitability in humans: a transcranial magnetic stimulation study. Ann Neurol 40: 367-378. 